\title{
A mathematical theory of stochastic microlensing. II. Random images, shear, and the Kac-Rice formula
}

\author{
A. O. Petters, ${ }^{1,2, a)}$ B. Rider, ${ }^{3, b)}$ and A. M. Teguia ${ }^{1, c)}$ \\ ${ }^{1}$ Department of Mathematics, Duke University, Science Drive, Durham, North Carolina \\ 27708, USA \\ ${ }^{2}$ Department of Physics, Duke University, Science Drive, Durham, North Carolina 27708, \\ USA \\ ${ }^{3}$ Department of Mathematics, University of Colorado at Boulder, Campus Box 395 \\ Boulder, Colorado 80309, USA
}

(Received 31 July 2008; accepted 3 November 2009; published online 15 December 2009)

\begin{abstract}
Continuing our development of a mathematical theory of stochastic microlensing, we study the random shear and expected number of random lensed images of different types. In particular, we characterize the first three leading terms in the asymptotic expression of the joint probability density function (pdf) of the random shear tensor due to point masses in the limit of an infinite number of stars. Up to this order, the pdf depends on the magnitude of the shear tensor, the optical depth, and the mean number of stars through a combination of radial position and the star's mass. As a consequence, the pdf's of the shear components are seen to converge, in the limit of an infinite number of stars, to shifted Cauchy distributions, which shows that the shear components have heavy tails in that limit. The asymptotic pdf of the shear magnitude in the limit of an infinite number of stars is also presented. All the results on the random microlensing shear are given for a general point in the lens plane. Extending to the general random distributions (not necessarily uniform) of the lenses, we employ the Kac-Rice formula and Morse theory to deduce general formulas for the expected total number of images and the expected number of saddle images. We further generalize these results by considering random sources defined on a countable compact covering of the light source plane. This is done to introduce the notion of global expected number of positive parity images due to a general lensing map. Applying the result to microlensing, we calculate the asymptotic global expected number of minimum images in the limit of an infinite number of stars, where the stars are uniformly distributed. This global expectation is bounded, while the global expected number of images and the global expected number of saddle images diverge as the order of the number of stars. () 2009 American Institute of Physics. [doi:10.1063/1.3267859]
\end{abstract}

\section{INTRODUCTION}

We started an analytical study of stochastic microlensing in Paper $\mathrm{I}^{1}$ where we derived stochastic properties of the random time delay function, lensing map, and bending angle. We continue this work here by investigating the expected number of critical points (lensed images) of the time delay function or, equivalently, the expected number of solutions of the random lens equation.

There is a fair amount of literature dealing with the nonrandom number of images produced by a microlensing situation. One of the first works on this subject showed that a fixed source

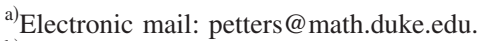

${ }^{b}$ Electronic mail: brider@euclid.colorado.edu.

${ }^{c)}$ Electronic mail: alberto@math.duke.edu.
} 
lensed by a single star can produce two distinct images. ${ }^{2}$ This result was generalized to any finite number of stars for both single and multiple plane lensing, with results on sharp lower bounds and counting formulas of the total number of images, the number of minimum images, number of saddles images, and number of maximum images ${ }^{3-5}$ (see Chaps. 11 and 12 in Ref. 6). Sharp upper bounds on the total number of images have also been obtained (see Refs. 7 and 8). The case of stars with random positions was later examined using semianalytical methods, yielding asymptotic formulas in the limit of an infinite number of stars for the expected number of minima (e.g., Refs. 9 and 10). However, these results are limited to first order approximations and are not applicable to general random distributions of the star positions or source positions.

In the current paper, we begin by deriving the first three terms in the asymptotic expansion of the microlensing shear tensor's probability density function (pdf) in the limit of an infinite number of stars. These results show that in such a limit, the marginal pdf's of the components of the random shear tensor converge in the sense of distributions to shifted Cauchy densities. We also show that the third term in this asymptotic pdf of the shear tensor depends on the square of the magnitude of the shear due only to stars, the optical depth, and the mean number of stars. The asymptotic pdf of the magnitude of shear is determined as well.

Next, we derive for a lensing map (not necessarily due to microlensing), a Kac-Rice type formula for the expected number of positive parity lensed images (i.e., minimum and maximum images) lying in a compact region of the lens plane. This formula can be applied in a large number of random lensing scenarios and is obtained using the Kac-Rice technique for computing the expected number of zeros of random mappings (e.g., Refs. 11-17). We then combine this formula and results from Morse theory to obtain additional general formulas for the expected total number of images and the expected number of saddle images.

This paper then concludes by applying the aforementioned Kac-Rice formula and the asymptotic joint pdf of the microlensing shear tensor to microlensing due to point masses with continuous matter and shear. Here, we focus our attention on the "global expectation" of the number of minimum images (which will be defined later). We consider the minimum images since numerical simulations have shown that they are significant contributors to high magnification events in microlensing light curves (e.g., Ref. 9).

The outline of this paper is as follows. The basic lensing framework is given in Sec. II. We obtain the asymptotic joint pdf for the microlensing shear components in Sec. III. In Sec. IV, we derive the general formulas for the expected total number of positive parity images, the expected total number of images, and the expected total number of saddle images. In the same section, we define the global expectation and obtain an integral formula for the global expected number of positive parity images. Section V presents applications of these general formulas, along with the results on the random microlensing shear, to determine a global expected number of minimum images in stochastic microlensing.

\section{BASICS}

A general gravitational lens system is governed by its time delay function

$$
T_{y}(x)=\frac{|x-y|^{2}}{2}-\psi(x)
$$

and lensing map

$$
\eta(x)=x-\nabla \psi(x)
$$

where $y$ is the light source's position and $\psi$ is the gravitational lens potential (Ref. 6, Chap. 6). A lensed image of a light source at $y$ is a critical point $x$ of $T_{y}$, that is, a solution $x$ in $\mathbb{R}^{2}$ of the equation $\nabla T_{y}(x)=\mathbf{0}$. It immediately follows that lensed images are solutions $x$ in $\mathbb{R}^{2}$ of the equation,

$$
\eta(x)=y,
$$


which is called the lens equation. When the potential $\psi$ or the source position $y$ becomes random, the lens equation has a random number of solutions (lensed images).

A lensed image $x$ is nondegenerate (degenerate) if $x$ is a nondegenerate (degenerate) critical point of $T_{y}$. Using the Morse lemma (see Ref. 18), we can classify a nondegenerate light ray as either a local minimum (if $x$ has index 0), a local saddle (if $x$ has index 1), or a local maximum (if $x$ has index 2) (see Ref. 6, p. 178 for more information). The set of positive parity lensed images consists of all the minimum and maximum lensed images.

In stochastic microlensing, we consider a gravitational field due to a collection of $g$ stars at positions $\xi_{1}, \ldots, \xi_{g}$ that are independent and identically distributed over a region of the lens plane. The resulting gravitational lens potential $\psi_{g}$, time delay function $T_{y, g}$ at $y$, and lensing map $\eta_{g}$ become random and are given by

$$
\begin{gathered}
\psi_{g}(x)=\frac{\kappa_{c}}{2}|x|^{2}-\frac{\gamma}{2}\left(x_{1}^{2}-x_{2}^{2}\right)+\sum_{j=1}^{g} m_{j} \log \left|x-\xi_{j}\right|, \\
T_{y, g}(x)=\frac{1}{2}|x-y|^{2}-\frac{\kappa_{c}}{2}|x|^{2}+\frac{\gamma}{2}\left(x_{1}^{2}-x_{2}^{2}\right)-\sum_{j=1}^{g} m_{j} \log \left|x-\xi_{j}\right|, \\
\eta_{g}(x)=\left(\left(1-\kappa_{c}+\gamma\right) x_{1},\left(1-\kappa_{c}-\gamma\right) x_{2}\right)-\sum_{j=1}^{g} m_{j} \frac{x-\xi}{|x-\xi|^{2}},
\end{gathered}
$$

where $x=\left(x_{1}, x_{2}\right)$ is in $L=\mathbb{R}^{2}-\left\{\xi_{1}, \ldots, \xi_{g}\right\}$ (lens plane). Unless stated to the contrary, the light source positions $y$, the continuous matter density $\kappa_{c}$, external shear $\gamma$, masses $m_{j}$, and number of stars $g$ are assumed fixed. The star positions $\xi_{j}=\left(U_{j}, V_{j}\right)$ are random vectors. We shall consider the case of random light source positions in Secs. IV and V.

In Secs. III and V, we shall work under the following assumptions about microlensing:

- Cartesian coordinates $(u, v)$ are assumed in the plane $\mathbb{R}^{2}$ with $\mathbf{0}$ as the origin.

- All masses are assumed equal: $m_{1}=m_{2}=\cdots=m_{g} \equiv m \neq 0$.

- The star positions $\xi_{i}=\left(U_{i}, V_{i}\right)$ are independent and uniformly distributed in the disk $B(\mathbf{0}, R)$ of radius $R$ centered at $\mathbf{0}$, that is,

$$
\left(U_{i}, V_{i}\right) \sim \operatorname{Unif}\left\{(u, v) \in \mathbb{R}^{2}:|u|^{2}+|v|^{2} \leq R^{2}\right\}, \quad i=1, \ldots, g .
$$

- The quantities $R$ and $g$ are related by the following physical formula for the optical depth $\kappa_{*}$ in point masses (stars):

$$
\kappa_{*}=\frac{m g}{R^{2}}
$$

The quantity $\kappa_{*}$ is fixed. For $x \in \mathbb{R}^{2}$, with $0 \leq|x|<R$, we shall call the combination $\kappa_{*}|x|^{2} / m$ the mean number of stars within the disk of radius $|x|$ about the origin.

Remarks: The assumptions stated above provide a good approximation for several astrophysical scenarios. Note that another lensing model used in literature is the elliptical lens model (see Ref. 6, pp. 101-108 for more on lens models).

\section{RANDOM SHEAR DUE TO POINT MASSES}

The shear at $x$ is a gravitational tug of stars on the light ray through $x$. The two components of the shear tensor at $x=\left(x_{1}, x_{2}\right) \in L$ due to point masses (stars) are denoted by $\Gamma_{g}^{*}(x)$ $=\left(\Gamma_{1, g}^{*}(x), \Gamma_{2, g}^{*}(x)\right)$, with components as follows (see Ref. 6, p. 96): 


$$
\Gamma_{1, g}^{*}(x)=\sum_{j=1}^{g} \frac{m\left(\left(U_{j}-x_{1}\right)^{2}-\left(V_{j}-x_{2}\right)^{2}\right)}{\left[\left(U_{j}-x_{1}\right)^{2}+\left(V_{j}-x_{2}\right)^{2}\right]^{2}} \quad \text { and } \quad \Gamma_{2, g}^{*}(x)=\sum_{j=1}^{g} \frac{2 m\left(U_{j}-x_{1}\right)\left(V_{j}-x_{2}\right)}{\left[\left(U_{j}-x_{1}\right)^{2}+\left(V_{j}-x_{2}\right)^{2}\right]^{2}} .
$$

We study the asymptotic behavior of the random shear tensor $\left(\Gamma_{1, g}^{*}, \Gamma_{2, g}^{*}\right)$ due to the point masses by obtaining several leading terms of the asymptotic joint pdf of its components in the limit $g \rightarrow \infty$. To do so, we assume (for the remainder of the paper) that the random shear tensor has an absolutely continuous distribution function. We then obtain the following:

Theorem 1: Let $x=\left(x_{1}, x_{2}\right) \in \mathbb{R}^{2}$ with $|x|<R$ be fixed. The joint $p d f f_{\Gamma_{1, g}^{*}(x), \Gamma_{2, g}^{*}(x)}$ of the pair $\left(\Gamma_{1, g}^{*}(x), \Gamma_{2, g}^{*}(x)\right)$ is given in the limit $g \rightarrow \infty$ by

$$
f_{\Gamma_{1, g}^{*}(x), \Gamma_{2, g}^{*}(x)}(z, w)=\frac{\kappa_{*}}{2 \pi\left(\kappa_{*}^{2}+\Gamma^{2}\right)^{3 / 2}}\left[1+\frac{H_{1}(\Gamma)}{g}+\frac{H_{2}(\Gamma ;|x|)}{g^{2}}\right]+O\left(g^{-3}\right),
$$

where $(z, w)$ denotes the possible values of $\left(\Gamma_{1, g}^{*}(x), \Gamma_{2, g}^{*}(x)\right), \Gamma=\sqrt{z^{2}+w^{2}}$, denotes the possible values of the shear tensor's magnitude, and

$$
\begin{gathered}
H_{1}(z, w)=H_{1}(\Gamma)=\kappa_{*}^{2} \frac{9 \Gamma^{2}-6 \kappa_{*}^{2}}{4\left(\kappa_{*}^{2}+\Gamma^{2}\right)^{2}}, \\
H_{2}(z, w ;|x|)=H_{2}(\Gamma ;|x|)=\frac{\kappa_{*}|x|^{2}}{m} \frac{\kappa_{*}^{2}\left(6 \kappa_{*}^{2}-9 \Gamma^{2}\right)}{2\left(\kappa_{*}^{2}+\Gamma^{2}\right)^{2}}-\frac{\kappa_{*}^{2}\left(8 \kappa_{*}^{4}-24 \kappa_{*}^{2} \Gamma^{2}+3 \Gamma^{4}\right)}{4\left(\kappa_{*}^{2}+\Gamma^{2}\right)^{3}} \\
+\frac{15 \kappa_{*}^{4}\left(8 \kappa_{*}^{4}-40 \kappa_{*}^{2} \Gamma^{2}+15 \Gamma^{4}\right)}{32\left(\kappa_{*}^{2}+\Gamma^{2}\right)^{4}} .
\end{gathered}
$$

The pdf at this order is a function of the magnitude of the shear due only to stars, optical depth $\kappa_{*}$ and the mean number of stars within the disk of radius $|x|$ about the origin.

The marginals of $f_{\Gamma_{1, g}^{*}(x), \Gamma_{2, g}^{*}(x)}$ are

$$
f_{\Gamma_{i, g}(x)}\left(a_{i}\right)=\frac{\kappa_{*}}{\pi\left(\kappa_{*}^{2}+a_{i}^{2}\right)}\left[1+\frac{K_{1}\left(a_{i}^{2}\right)}{g}+\frac{K_{2}\left(a_{i}^{2} ;|x|\right)}{g^{2}}\right]+O\left(g^{-3}\right), \quad i=1,2,
$$

where $a_{1}=z, a_{2}=w$ and

$$
\begin{gathered}
K_{1}(h)=\kappa_{*}^{2} \frac{3 h-\kappa_{*}^{2}}{2\left(\kappa_{*}^{2}+h\right)^{2}}, \\
K_{2}(h ;|x|)=\frac{\kappa_{*}|x|^{2}}{m} \frac{\kappa_{*}^{2}\left(\kappa_{*}^{2}-3 h\right)}{\left(\kappa_{*}^{2}+h\right)^{2}}-\frac{\kappa_{*}^{2}}{2} \frac{\kappa_{*}^{4}-6 \kappa_{*}^{2} h+h^{2}}{\left(\kappa_{*}^{2}+h\right)^{3}}+\frac{3 \kappa_{*}^{4}}{4} \frac{\kappa_{*}^{4}-10 \kappa_{*}^{2} h+5 h^{2}}{\left(\kappa_{*}^{2}+h\right)^{4}} .
\end{gathered}
$$

Proof: We set up coordinates in the same way as in the proof of Proposition 1 in Ref. 1. For the reader's convenience, we repeat the details. Let $x=\left(x_{1}, x_{2}\right) \in \mathbb{R}^{2}$ with $|x|<R$. Translate the rectangular coordinates $(u, v)$ so that its origin $\mathbf{0}$ moves to position $x$ and denote the resulting new coordinates by $\left(u^{\prime}, v^{\prime}\right)$ and its origin by $\mathbf{0}^{\prime}$. The old origin $\mathbf{0}$ in $\left(u^{\prime}, v^{\prime}\right)$ has coordinates $\left(u^{\prime}, v^{\prime}\right)$ $=-x$. Define $\omega_{0}$ to be the unique principal angle with $\cos \omega_{0}=x_{1} /|x|, \sin \omega_{0}=x_{2} /|x|$, and let $\omega$ $\equiv \omega_{0}+\pi$. Rotating the rectangular coordinates $\left(u^{\prime}, v^{\prime}\right)$ counterclockwise by angle $\omega$, we obtain new coordinates $\left(u^{\prime \prime}, v^{\prime \prime}\right)$ with origin $\mathbf{0}^{\prime \prime}=\mathbf{0}^{\prime}$. The old origin $\mathbf{0}$ now lies at position $\left(u^{\prime \prime}, v^{\prime \prime}\right)$ $=(|x|, 0) \equiv x^{\prime \prime}$ on the positive $u^{\prime \prime}$-axis. Let $(\theta, r)$ denote polar coordinates in the frame $\left(u^{\prime \prime}, v^{\prime \prime}\right)$.

Let $x=\left(x_{1}, x_{2}\right) \in \mathbb{R}^{2}$ with $|x|<R$ and set $u_{x}=u-x_{1}, v_{x}=v-x_{2}$, and $\mathfrak{r}_{x}^{2}=u_{x}^{2}+v_{x}^{2}$. The joint characteristic function of $\left(\Gamma_{1, g}^{*}(x), \Gamma_{2, g}^{*}(x)\right)$ is given by 


$$
\begin{aligned}
\left(\varphi_{\Gamma_{1, g}^{*}(x), \Gamma_{2, g}^{*}(x)}\left(t_{1}, t_{2}\right)\right)^{1 / g}= & \frac{1}{\pi R^{2}} \int_{B(\mathbf{0}, R)} \exp \left[i t_{1}\left(\frac{m\left(u_{x}^{2}-v_{x}^{2}\right)}{\mathfrak{r}_{x}^{4}}\right)+i t_{2}\left(\frac{2 m u_{x} v_{x}}{\mathfrak{r}_{x}^{4}}\right)\right] \mathrm{d} u \mathrm{~d} v \\
= & \frac{1}{\pi R^{2}} \int_{B(x, R-|x|)} \exp \left[i \frac{m}{\mathfrak{r}_{x}^{4}}\left(t_{1}, t_{2}\right) \cdot\left(u_{x}^{2}-v_{x}^{2}, 2 u_{x} v_{x}\right)\right] \mathrm{d} u \mathrm{~d} v \\
& +\frac{1}{\pi R^{2}} \int_{B(\mathbf{0}, R) B(x, R-|x|)} \exp \left[i \frac{m}{\mathfrak{r}_{x}^{4}}\left(t_{1}, t_{2}\right) \cdot\left(u_{x}^{2}-v_{x}^{2}, 2 u_{x} v_{x}\right)\right] \mathrm{d} u \mathrm{~d} v \\
= & \frac{2}{R^{2}} \int_{0}^{R-|x|} r J_{0}\left(\frac{m t}{r^{2}}\right) \mathrm{d} r+\frac{1}{\pi R^{2}} \int_{\Omega} \exp \left[\frac{i m t \cos \left(2\left(\theta+\omega^{\prime}\right)\right)}{r^{2}}\right] \mathrm{d} \theta \mathrm{d} r,
\end{aligned}
$$

with $t=\sqrt{t_{1}^{2}+t_{2}^{2}}, 2 \omega^{\prime}=2 \omega+\phi, \quad \phi=\arctan \left(t_{1} / t_{2}\right), J_{0}$ is the zero Bessel function.

Now, with $\nu=m t$, we get

$$
\begin{aligned}
\frac{2}{R^{2}} \int_{0}^{R-|x|} r J_{0}\left(\frac{\nu}{r^{2}}\right) \mathrm{d} r & =\frac{\nu}{R^{2}}\left[\frac{(R-|x|)^{2}}{\nu}{ }_{1} F_{2}\left(-\frac{1}{2} ; \frac{1}{2}, 1 ; \frac{-\nu^{2}}{4(R-|x|)^{4}}\right)-1\right] \\
& =-\frac{\nu}{R^{2}}+\frac{(R-|x|)^{2}}{R^{2}}\left[1+\frac{\nu^{2}}{4(R-|x|)^{4}}+O\left(R^{-8}\right)\right],
\end{aligned}
$$

where ${ }_{1} F_{2}$ is the hypergeometric function.

We use Taylor series expansion and term-by-term integration to write the second integral in Eq. (5) as

$$
\frac{1}{\pi R^{2}} \int_{\Omega} \exp \left[\frac{i \nu \cos \left(2\left(\theta+\omega^{\prime}\right)\right)}{r^{2}}\right] \mathrm{d} u \mathrm{~d} v=\frac{1}{\pi R^{2}}\left[\pi\left(2|x| R-|x|^{2}\right)+\sum_{n=1}^{\infty} \frac{(i \nu)^{n}}{n !} I_{n}\right]
$$

where, for integers $n \geq 1$,

$$
\begin{aligned}
I_{n} & =\int_{R-|x|}^{R+|x|} r \int_{-f(r)}^{f(r)} \frac{\cos ^{n}\left(2\left(\theta+\omega^{\prime}\right)\right)}{r^{2 n}} \mathrm{~d} \theta \mathrm{d} r \\
& =\int_{R-|x|}^{R+|x|} \frac{1}{r^{2 n-1}} \int_{-f(r)}^{f(r)}\left(a_{n}+\sum_{j=0}^{b_{n}} c_{n, j} \cos \left[2(n-2 j)\left(\theta+\omega^{\prime}\right)\right]\right) \mathrm{d} \theta \mathrm{d} r \\
& =\int_{R-|x|}^{R+|x|} \frac{1}{r^{2 n-1}}\left(2 a_{n} f(r)+\sum_{j=1}^{b_{n}} \frac{c_{n, j} \cos \left[2(n-2 j) \omega^{\prime}\right]}{n-2 j} \sin [2(n-2 j) f(r)]\right) \mathrm{d} r+\frac{c_{n, 0} \cos \left[2 n \omega^{\prime}\right]}{n} \Im_{2 n},
\end{aligned}
$$

where

$$
\begin{array}{r}
\mathfrak{I}_{n} \\
\quad=\int_{R-|x|}^{R+|x|} \frac{\sin [n f(r)]}{r^{n-1}} \mathrm{~d} r,
\end{array}
$$

and the coefficients $a_{n}, b_{n}$, and $c_{n, j}^{\prime}$ 's are obtained by finding the Fourier series of $\cos ^{n} \theta$. We will prove in the Appendix that $\mathfrak{I}_{n}=0$ for every integer $n \geq 2$. We then obtain

$$
\begin{gathered}
I_{1}=0, \\
I_{2}=\int_{R-|x|}^{R+|x|} \frac{f(r)}{r^{3}} \mathrm{~d} r=\frac{\pi}{2} \frac{|x|(2 R+|x|)}{\left(R^{2}-|x|^{2}\right)^{2}},
\end{gathered}
$$




$$
\begin{gathered}
I_{3}=\frac{3 \cos 2 \omega^{\prime}}{2} \int_{R-|x|}^{R+|x|} \frac{\cos (f(r)) \sin (f(r))}{r^{5}} \mathrm{~d} r=-\frac{3 \cos 2 \omega^{\prime}}{2} \frac{\pi|x|^{2} R^{2}}{2\left(R^{2}-|x|^{2}\right)^{4}}=O\left(R^{-6}\right), \\
\left|I_{n}\right| \leq \int_{R-|x|}^{R+|x|} \frac{4 \pi+1}{r^{2 n-1}} \mathrm{~d} r \leq \frac{2|x|(4 \pi+1)}{(R-|x|)^{2 n-1}}=O\left(R^{-2 n+1}\right) \quad \text { for } n \geq 0 .
\end{gathered}
$$

Therefore,

$$
\frac{1}{\pi R^{2}} \int_{\Omega} \exp \left[\frac{i \nu \cos \left(2\left(\theta+\omega^{\prime}\right)\right)}{r^{2}}\right] \mathrm{d} u \mathrm{~d} v=\frac{2|x|}{R}-\frac{|x|^{2}}{R^{2}}-\frac{|x| \nu^{2}}{2 R^{5}}-\frac{|x|^{2} \nu^{2}}{4 R^{6}}-\frac{|x|^{3} \nu^{2}}{R^{7}}+O\left(R^{-8}\right) .
$$

We now substitute Eqs. (6) and (7) into Eq. (5) to obtain

$$
\left(\varphi_{\Gamma_{1, g}^{*}(x), \Gamma_{2, g}^{*}(x)}\left(t_{1}, t_{2}\right)\right)^{1 / g}=1-\frac{\nu}{R^{2}}+\frac{\nu^{2}}{4 R^{4}}+\frac{|x|^{2} \nu^{2}}{2 R^{6}}+O\left(R^{-8}\right)=1-\frac{\kappa_{*} t}{g}+\frac{\kappa_{*}^{2} t^{2}}{4 g^{2}}+\frac{|x|^{2} \kappa_{*}^{3} t^{2}}{2 m g^{3}}+O\left(g^{-4}\right) .
$$

An asymptotic expansion of the characteristic function is then derived apply the logarithmic function of both sides of the above equation,

$$
\begin{aligned}
\log \left(\varphi_{\Gamma_{1, g}^{*}(x), \Gamma_{2, g}^{*}(x)}\left(t_{1}, t_{2}\right)\right) & =g \log \left[1-\frac{\kappa_{*} t}{g}+\frac{\kappa_{*}^{2} t^{2}}{4 g^{2}}+\frac{|x|^{2} \kappa_{*}^{3} t^{2}}{2 m g^{3}}+O\left(g^{-4}\right)\right] \\
& =-\nu_{1}-\frac{\nu_{1}^{2}}{4 g}+\left(\frac{|x|^{2} \kappa_{*} \nu_{1}^{2}}{2 m}-\frac{\nu_{1}^{3}}{12}\right) \frac{1}{g^{2}}+O\left(g^{-3}\right),
\end{aligned}
$$

thus,

$$
\begin{aligned}
e^{\nu_{1}} \varphi_{\Gamma_{1, g}^{*}(x), \Gamma_{2, g}^{*}(x)}\left(t_{1}, t_{2}\right) & =\exp \left[-\frac{\nu_{1}^{2}}{4 g}+\left(\frac{|x|^{2} \kappa_{*} \nu_{1}^{2}}{2 m}-\frac{\nu_{1}^{3}}{12}\right) \frac{1}{g^{2}}+O\left(g^{-3}\right)\right] \\
& =1-\frac{\nu_{1}^{2}}{4 g}+\left(\frac{|x|^{2} \kappa_{*} \nu_{1}^{2}}{2 m}-\frac{\nu_{1}^{3}}{12}+\frac{\nu_{1}^{4}}{32}\right) \frac{1}{g^{2}}+O\left(g^{-3}\right) .
\end{aligned}
$$

Hence

$$
\varphi_{\Gamma_{1, g}^{*}(x), \Gamma_{2, g}^{*}(x)}\left(t_{1}, t_{2}\right)=e^{\nu_{1}}\left[1-\frac{\nu_{1}^{2}}{4 g}+\left(\frac{|x|^{2} \kappa_{*} \nu_{1}^{2}}{2 m}-\frac{\nu_{1}^{3}}{12}+\frac{\nu_{1}^{4}}{32}\right) \frac{1}{g^{2}}\right]+O\left(g^{-3}\right) .
$$

By assumption, the joint pdf $f_{\Gamma_{1, g}^{*}(x), \Gamma_{2, g}^{*}(x)}$ of $\left(\Gamma_{1, g}^{*}(x), \Gamma_{2, g}^{*}(x)\right)$ exists, i.e., the inverse Fourier transform (IFT) of the left hand side (LHS) of Eq. (8) exists. Consequently, so does the IFT of the right hand side (RHS) of Eq. (8). The latter can be computed as the sum of the IFT of functions of the form $p(g) \times q\left(t_{1}, t_{2}\right)$, where $p(g)$ is a function of $g$ and $q\left(t_{1}, t_{2}\right)$ is independent of $g$ and is integrable.

Let

$$
\varphi_{g}\left(t_{1}, t_{2}\right)=e^{-\nu_{1}}\left[1-\frac{\nu_{1}^{2}}{4 g}+\left(\frac{|x|^{2} \kappa_{*} \nu_{1}^{2}}{2 m}-\frac{\nu_{1}^{3}}{12}+\frac{\nu_{1}^{4}}{32}\right) \frac{1}{g^{2}}\right] .
$$

We have

$$
f_{\Gamma_{1, g}^{*}(x), \Gamma_{2, g}^{*}(x)}(z, w)=\left(\operatorname{IFT}\left(\varphi_{g}\right)\right)(z, w)+O\left(g^{-3}\right) .
$$

Here, $\operatorname{IFT}\left(\varphi_{g}\right)$ is the IFT of $\varphi_{g}$ and can be obtained by computing integrals of the form 


$$
\begin{aligned}
& \frac{1}{(2 \pi)^{2}} \int_{\mathrm{R}^{2}} e^{-\kappa_{*} \sqrt{t_{1}^{2}+t_{2}^{2}}\left(t_{1}^{2}+t_{2}^{2}\right)^{n / 2} e^{-i\left(t_{1} z+t_{2} w\right)} \mathrm{d} t_{1} \mathrm{~d} t_{2}} \\
& =\frac{1}{2 \pi}\left(z^{2}+w^{2}\right)^{-n / 2-1} \int_{0}^{\infty} r^{n+1} e^{-\kappa_{*}\left(z^{2}+w^{2}\right)^{-1 / 2} r} J_{0}(r) \mathrm{d} r \\
& =\frac{1}{2 \pi} \kappa_{*}^{-n-2} \Gamma(n+2)_{2} F_{1}\left(\frac{n+2}{2}, \frac{n+3}{2} ; 1 ;-\frac{z^{2}+w^{2}}{\kappa_{*}^{2}}\right) .
\end{aligned}
$$

We then complete the proof of the first part of the theorem by using Eq. (9) for $n=0,2,3,4$. Now consider the following functions defined on $R$ :

$$
\begin{gathered}
h_{1}(z) \equiv f_{\Gamma_{1, g}^{*}(x), \Gamma_{2, g}^{*}(x)}(z, w) \text { for fixed } w \in \mathbb{R}, \\
h_{2}(w) \equiv f_{\Gamma_{1, g}^{*}(x), \Gamma_{2, g}^{*}(x)}(z, w) \text { for fixed } z \in \mathbb{R}, \\
k_{1}(z) \equiv \frac{\kappa_{*}}{2 \pi} \frac{1}{\left(\kappa_{*}^{2}+z^{2}+w^{2}\right)^{3 / 2}}\left[1+\frac{H_{1}(z, w)}{g}+\frac{H_{2}(z, w ;|x|)}{g^{2}}\right] \quad \text { for fixed } w \in \mathbb{R}, \\
k_{2}(w) \equiv \frac{\kappa_{*}}{2 \pi} \frac{1}{\left(\kappa_{*}^{2}+z^{2}+w^{2}\right)^{3 / 2}}\left[1+\frac{H_{1}(z, w)}{g}+\frac{H_{2}(z, w ;|x|)}{g^{2}}\right] \quad \text { for fixed } z \in \mathbb{R} .
\end{gathered}
$$

We know that $h_{1}, h_{2} \in L^{1}(\mathbb{R})$ since the random shear's pdf is integrable. We can then prove that $k_{1}, k_{2} \in L^{1}(\mathbb{R})$ by direct computation. Hence, the second part of the theorem follows by integration with respect to $w$ and $z$.

Remark: The leading function in the RHS of Eq. (2) becomes a pdf asymptotically in $g$. In particular, set

$$
\mathcal{F}_{g,|x|}(z, w)=\frac{\kappa_{*}}{2 \pi\left(\kappa_{*}^{2}+z^{2}+w^{2}\right)^{3 / 2}}\left[1+\frac{H_{1}(z, w)}{g}+\frac{H_{2}(z, w ;|x|)}{g^{2}}\right] .
$$

Then, for $g$ sufficiently large,

$$
\mathcal{F}_{g,|x|}(z, w) \geq 0
$$

In addition,

$$
\int_{\mathbb{R}^{2}} \mathcal{F}_{g,|x|}(z, w) \mathrm{d} z \mathrm{~d} w=1
$$

for all $g \geq 1$ and $x \in R^{2}$. An analogous result was found in Ref. 1 for the random lensing map (see the discussion after Corollary 10).

Corollary 2: For any $x \in \mathbb{R}^{2}$, the pair $\left(\Gamma_{1, g}^{*}(x), \Gamma_{2, g}^{*}(x)\right)$ converges in the sense of distributions to the pair $\left(\Gamma_{1, \infty}^{*}, \Gamma_{2, \infty}^{*}\right)$ with joint density given by

$$
f_{\Gamma_{1, \infty}^{*}, \Gamma_{2, \infty}^{*}}(z, w)=\frac{\kappa_{*}}{2 \pi} \frac{1}{\left[\kappa_{*}^{2}+z^{2}+w^{2}\right]^{3 / 2}}
$$

as $g \rightarrow \infty$. Note that $f_{\Gamma_{1, \infty}^{*}, \Gamma_{2, \infty}^{*}}$ is a (stretched) bivariate Cauchy distribution with marginals,

$$
f_{\Gamma_{1, \infty}^{*}}(z)=\frac{\kappa_{*}}{\pi\left(\kappa_{*}^{2}+z^{2}\right)} \quad \text { and } \quad f_{\Gamma_{2, \infty}^{*}}(w)=\frac{\kappa_{*}}{\pi\left(\kappa_{*}^{2}+w^{2}\right)} .
$$


Corollary 2 is consistent with the findings by Nityananda and Ostriker ${ }^{19}$ and Schneider, Ehlers, and Falco ${ }^{20}$ (see p. 329). Note that the corollary shows that the random shear components due to point masses have heavy tails as $g \rightarrow \infty$, an important point we have not seen emphasized in literature.

Corollary 3: Let $x \in \mathbb{R}^{2}$ with $|x|<R$ be fixed and consider the magnitude of the shear at $x$ due only to stars, namely, $\Gamma_{g}^{*}(x)=\sqrt{\left(\Gamma_{1, g}^{*}(x)\right)^{2}+\left(\Gamma_{1, g}^{*}(x)\right)^{2}}$. The pdf $f_{\Gamma_{g}^{*}(x)}$ of $\Gamma_{g}^{*}(x)$ is given in the limit $g \rightarrow \infty$ by

$$
f_{\Gamma_{g}^{*}(x)}(\Gamma)=\frac{\kappa_{*} \Gamma}{\left(\kappa_{*}^{2}+\Gamma^{2}\right)^{3 / 2}}\left[1+\frac{H_{1}(\Gamma)}{g}+\frac{H_{2}(\Gamma ;|x|)}{g^{2}}\right]+O\left(g^{-3}\right) .
$$

Here, $\Gamma$ denotes the possible values of $\Gamma_{g}^{*}(x)$.

Proof: We use the change in variable

$$
\left\{\begin{array}{l}
\Gamma_{1, g}^{*}(x)=\Gamma_{g}^{*}(x) \cos \Theta(x) \\
\Gamma_{2, g}^{*}(x)=\Gamma_{g}^{*}(x) \sin \Theta(x)
\end{array}\right.
$$

and obtain

$$
\begin{aligned}
f_{\Gamma_{g}^{*}(x), \Theta(x)}(\Gamma, \theta) & =\Gamma f_{\Gamma_{1, g}^{*}(x), \Gamma_{2, g}^{*}(x)}(\Gamma \cos \theta, \Gamma \sin \theta) \\
& =\frac{\Gamma}{2 \pi} \frac{\kappa_{*}}{\left(\kappa_{*}^{2}+\Gamma^{2}\right)^{3 / 2}}\left[1+\frac{H_{1}(\Gamma)}{g}+\frac{H_{2}(\Gamma ;|x|)}{g^{2}}\right]+O\left(g^{-3}\right) .
\end{aligned}
$$

We can then integrate the equation above with respect to $\theta$ from 0 to $2 \pi$ and obtain the result stated in the corollary.

\section{EXPECTED NUMBER OF IMAGES: GENERAL CASE}

In this section, we are not restricting ourselves to the point-mass model as was the case in Sec. III. We shall determine results for the expected number of images for a general lensing map.

\section{A. Expected number of positive parity images: General case and the Kac-Rice formula}

We work under the following broad, natural assumptions and notation:

- The gravitational field potential $\psi$ is an isolated potential with a finite number of singularities, all of which are infinite (see Ref. 6, p. 424 for more on isolated potentials).

- The potential $\psi$ and lensing map $\eta$ are smooth, except on a set of measure zero.

- Notation:

(a) For a set $A$, let

$$
\mathbf{1}_{A}(x)= \begin{cases}1 & \text { if } x \in A \\ 0 & \text { if } x \notin A .\end{cases}
$$

(b) Set $G(x)=\operatorname{det}(\operatorname{Jac} \eta)(x), A=(0, \infty)$, and $\mathcal{G}_{A}=\left\{\nu \in \mathbb{R}^{2}: G(\nu) \in A\right\}$, where $\eta$ a general lensing map.

(c) Let $N_{+}=N_{\min }+N_{\max }$, which is the total number of maximum and minimum images.

(d) Let $N_{+}(D, y)=$ total number of positive parity images (minima and maxima) lying in a subset $D$ of the lens plane for fixed source position $y$.

The determinant of the Jacobian of the general lensing map $\eta$ is given by (see Ref. 6, p. 182)

$$
\operatorname{det}(\operatorname{Jac} \eta)(x)=(1-\kappa(x))^{2}-\Gamma^{2}(x)=(1-\kappa(x))^{2}-\left[\Gamma_{1}^{2}(x)+\Gamma_{2}^{2}(x)\right] .
$$

Here, $\Gamma_{1}$ and $\Gamma_{2}$ are the shear components of the general lensing map $\eta$ and are defined by 


$$
\Gamma_{1}(x)=\frac{\psi_{11}(x)-\psi_{22}(x)}{2} \text { and } \quad \Gamma_{2}(x)=\psi_{12}(x)
$$

and $\kappa(x)$ is the convergence defined by

$$
\kappa(x)=\frac{\psi_{11}(x)+\psi_{22}(x)}{2}
$$

where $\psi_{i j}=\partial^{2} \psi / \partial x_{i} \partial x_{j}$.

The main result is as follows.

Theorem 4: (Expected number of positive parity images) Let $D \subset \mathbb{R}^{2}$ be a closed disk. Assume the following:

(1) $P[\{x \in D: \eta(x)=y$ and $\operatorname{det}(\operatorname{Jac} \eta)(x)=0\}]=0$.

(2) $\quad \kappa$ is bounded on L (lens plane).

Then, for a test function $\rho: \mathbb{R}^{2} \rightarrow \mathbb{R}$ with compact support, we have

$$
\int_{\mathbb{R}^{2}} \rho(y) E\left[N_{+}(D, y)\right] \mathrm{d} y=\int_{\mathbb{R}^{2}} \rho(y) \int_{D} E\left[\operatorname{det}(\operatorname{Jac} \eta)(x) \mathbf{1}_{\mathcal{G}_{A}}(x) \mid \eta(x)=y\right] f_{\eta(x)}(y) \mathrm{d} x \mathrm{~d} y
$$

and, for almost all $y$ in $\mathrm{R}^{2}$, the following Kac-Rice formula holds for lensing:

$$
E\left[N_{+}(D, y)\right]=\int_{D} E\left[\left((1-\kappa(x))^{2}-\Gamma^{2}(x)\right) \mathbf{1}_{\mathcal{G}_{A}}(x) \mid \eta(x)=y\right] f_{\eta(x)}(y) \mathrm{d} x .
$$

Before we prove this theorem, it is important to note that it can be applied to a large range of lens models. Moreover, the singularities form a set of measure zero and so contribute a value of zero to these integrals (see Ref. 21, p. 20).

The first assumption of Theorem 4 requires that the random images are almost surely not critical points of the random lensing map. The second assumption holds for optical depth constant in $L$.

Proof: The proof follows, with some modifications, the proof of the Kac-Rice formula given by Azais and Wschebor in Ref. 12 (Theorem 2.1). A more general version of the Kac-Rice formula with a different proof is given by Adler and Taylor in Ref. 11. The main tool used in the proof below is Federer's coarea formula. ${ }^{22}$

We start by fixing the source position at $y$. By Assumption 1 and results in Refs. 3, 4, 7, and 8 , the random function $N_{+}(D, y)$ is uniformly bounded, except on sets in $\mathrm{R}^{2}$ of measure zero. Let $\rho: \mathbb{R}^{2} \rightarrow \mathbb{R}$ be a test function with compact support. Since $\eta$ is smooth almost everywhere in $\mathbb{R}^{2}$ and $\rho(y)$ is integrable, we can apply Federer's coarea formula (see Ref. 11, p. 303) and obtain

$$
\int_{\mathbb{R}^{2}} \rho(y) N_{+}(D, y) \mathrm{d} y=\int_{D}|\operatorname{det}(\operatorname{Jac} \eta)(x)| \rho(\eta(x)) \mathbf{1}_{\mathcal{G}_{A}}(x) \mathrm{d} x .
$$

Taking expectations on both sides of the above equation yields

$$
\int_{\mathbb{R}^{2}} \rho(y) E\left[N_{+}(D, y)\right] \mathrm{d} y=\int_{D} E\left[\operatorname{det}(\operatorname{Jac} \eta)(x) \rho(\eta(x)) \mathbf{1}_{\mathcal{G}_{A}}(x)\right] \mathrm{d} x .
$$

The LHS (and hence the RHS) of Eq. (12) is bounded and the absolute value in the RHS was dropped since we are only considering positive parity images. Using the basic result 


$$
E[U]=\int_{\mathrm{R}^{n}} E[U \mid \mathbf{V}=\mathbf{v}] f_{\mathbf{V}}(\mathbf{v}) \mathrm{d} \mathbf{v},
$$

where $U$ is a real-valued random variable, $\mathbf{V}$ is a $\mathbb{R}^{n}$-valued random variable, and $\mathbf{v} \in \mathbb{R}^{n}$, we obtain

$$
E\left[\operatorname{det}(\operatorname{Jac} \eta)(x) \rho(\eta(x)) \mathbf{1}_{\mathcal{G}_{A}}(x)\right]=\int_{\mathbb{R}^{2}} E\left[\operatorname{det}(\operatorname{Jac} \eta)(x) \rho(\eta(x)) \mathbf{1}_{\mathcal{G}_{A}}(x) \mid \eta(x)=y\right] f_{\eta(x)}(y) \mathrm{d} y .
$$

Note that by Eq. (12), the expectation $E\left[\operatorname{det}(\operatorname{Jac} \eta)(x) \rho(\eta(x)) \mathbf{1}_{\mathcal{G}_{A}}(x)\right]$ is bounded on $D$ except on subsets of measure zero. The above equation then implies that

$$
\int_{D} \int_{\mathrm{R}^{2}}\left|E\left[\operatorname{det}(\operatorname{Jac} \eta)(x) \rho(\eta(x)) \mathbf{1}_{\mathcal{G}_{A}}(x) \mid \eta(x)=y\right] f_{\eta(x)}(y)\right| \mathrm{d} y \mathrm{~d} x
$$

is finite. Therefore,

$$
\begin{aligned}
\int_{\mathbb{R}^{2}} \rho(y) E\left[N_{+}(D, y)\right] \mathrm{d} y & =\int_{D} \int_{\mathbb{R}^{2}} E\left[\operatorname{det}(\operatorname{Jac} \eta)(x) \rho(\eta(x)) \mathbf{1}_{\mathcal{G}_{A}}(x) \mid \eta(x)=y\right] f_{\eta(x)}(y) \mathrm{d} y \mathrm{~d} x \\
& =\int_{\mathbb{R}^{2}} \int_{D} E\left[\operatorname{det}(\operatorname{Jac} \eta)(x) \rho(\eta(x)) \mathbf{1}_{\mathcal{G}_{A}}(x) \mid \eta(x)=y\right] f_{\eta(x)}(y) \mathrm{d} x \mathrm{~d} y \\
& =\int_{\mathbb{R}^{2}} \int_{D} E\left[\operatorname{det}(\operatorname{Jac} \eta)(x) \rho(y) \mathbf{1}_{\mathcal{G}_{A}}(x) \mid \eta(x)=y\right] f_{\eta(x)}(y) \mathrm{d} x \mathrm{~d} y \\
& =\int_{\mathbb{R}^{2}} \rho(y) \int_{D} E\left[\operatorname{det}(\operatorname{Jac} \eta)(x) \mathbf{1}_{\mathcal{G}_{A}}(x) \mid \eta(x)=y\right] f_{\eta(x)}(y) \mathrm{d} x \mathrm{~d} y,
\end{aligned}
$$

where the second equality follows the Fubini's theorem, the third follows the condition $\eta(x)=y$, and the last one follows the fact that $\rho(y)$ is deterministic and independent of $x$. This proves the result in Eq. (10). Since the test function $\rho$ was arbitrary, Eq. (11) is valid for almost all $y \in \mathbb{R}^{2}$.

Remark: For the critical case, if $\kappa(x)=1$ for almost all (a.a.) $x \in L$, then $G(x) \notin A$ for a.a. $x$ $\in L$, so $\mathbf{1}_{\mathcal{G}_{A}}(x)=0$ for a.a. $x \in L$. Hence, $E\left(N_{+}(D, y)\right)=0$. This is consistent with results in Ref. 6 .

\section{B. Expected number of images and saddle images: General case}

We can modify Theorem 4 to obtain a formula for the expected total number of images and the expected number of saddle images lying in $D$. However, it is simpler to use results from Morse theory contained in the following theorem (see Refs. 3, 4, and 6, Theorem 11.4, p. 424):

Theorem 5: (Ref. 6) Let $T$ and $\eta$ be, respectively, a single-plane time delay family and lensing map induced by an isolated gravitational lens potential $\psi$. Suppose that $\psi$ has $g$ singularities, all of which are infinite singularities. Then, the total number of lensed images of a light source at a noncaustic point $y$ is finite and the following holds:

(1) $\quad N_{\text {min }} \geq 1, \quad N_{\text {sad }} \geq g+N_{\text {max }}, \quad N_{\text {sad }} \geq N_{\min }+g-1, \quad N_{+}=N_{\text {sad }}-g+1$.

(2) $N_{\text {tot }}=2 N_{+}+g-1=2 N_{\text {sad }}-g+1, \quad N_{\text {tot }} \geq g+1$.

For a generalization of Theorem 5 to the case when the potential is not isolated, see Ref. 6, p. 422. We can apply Theorems 5 and 4 to obtain formulas for the expected total number of images and expected number of saddle images.

Corollary 6: Let $D$ be a closed disk whose interior contains the $g$ singularities of $\psi$. Then, under the assumptions of Theorem 4, the following holds for almost all y in $\mathrm{R}^{2}$ : 


$$
\begin{aligned}
& E\left[N_{\mathrm{tot}}(D, y)\right]=2 \int_{D} E\left[\left((1-\kappa(x))^{2}-\Gamma^{2}(x)\right) \mathbf{1}_{\mathcal{G}_{A}}(x) \mid \eta(x)=y\right] f_{\eta(x)}(y) \mathrm{d} x+g-1, \\
& E\left[N_{\mathrm{sad}}(D, y)\right]=\int_{D} E\left[\left((1-\kappa(x))^{2}-\Gamma^{2}(x)\right) \mathbf{1}_{\mathcal{G}_{A}}(x) \mid \eta(x)=y\right] f_{\eta(x)}(y) \mathrm{d} x+g-1,
\end{aligned}
$$

with self-evident notation.

Proof: Let $\xi_{1}, \ldots, \xi_{g}$ be the positions of the singularities. Choose $\epsilon_{0}$ small enough so that no image lies inside $B\left(\xi_{j}, \epsilon_{0}\right)$ for $j=1, \ldots, g$ and $B\left(\xi_{j}, \epsilon_{0}\right) \cap B\left(\xi_{i}, \epsilon_{0}\right)=\emptyset$ for all $i \neq j$. This is possible since the total number of images is bounded by

$$
g+1 \leq N_{\text {tot }} \leq 5 g-5
$$

(see Refs. 3 and 4 for the lower bound and Refs. 7 and 8 for the upper bound).

Set $D^{\prime}=D \backslash \cup_{j=1}^{g} B\left(\xi_{j}, \epsilon_{0}\right)$. The time delay function $T_{y}$ associated with $\psi$ generically satisfies Morse boundary condition A relative to $D^{\prime}$ (see Ref. 6, p. 403). We can then apply Theorem 5 to $D^{\prime}$ and obtain

$$
N_{\text {tot }}^{\prime}=2 N_{+}^{\prime}+g-1=2 N_{\text {sad }}^{\prime}-g+1 \text {, }
$$

where quantities with "prime" are related to $D$ '.

Equation (14) is an equality of random variables and holds for almost all source positions $y$ in $\mathrm{R}^{2}$. Therefore, we can take the expectations and obtain

$$
E\left[N_{\mathrm{tot}}^{\prime}\right]=2 E\left[N_{+}^{\prime}\right]+g-1=2 E\left[N_{\mathrm{sad}}^{\prime}\right]-g+1 .
$$

However, by construction,

$$
N_{\text {tot }}(D, y)=N_{\text {tot }}^{\prime}, \quad N_{+}(D, y)=N_{+}^{\prime}, \quad \text { and } \quad N_{\text {sad }}(D, y)=N_{\text {sad }}^{\prime} .
$$

The results then follow from Theorem 4.

Remark: In Corollary 6, the expected total number of images and number of saddles images both depend on the topology of the region $D \cap L$ since $g$ is its first Betti number.

\section{Global expectation: General case}

The expectations we shall study are actually generalizations of the expression $E\left[N_{+}(D, y)\right]$ in Eq. (11) since the source position is not known in the physical systems of interest to us (e.g., microlensing). Intuitively speaking, the quantity $E\left[N_{+}(D, y)\right]$ incorporates averaging with respect to the random components of the lensing map $\eta$ for a fixed source position $y$. We shall extend this to averaging over random source positions in a fixed compact set with positive measure in the light source plane and then generalize further to "averaging" over a family of such compact sets with positive measure. When all these averages are carried out, we refer to the result as a global expectation. We now make these ideas precise.

The first extension of $E\left[N_{+}(D, y)\right]$ is to consider a light source position in $\mathrm{R}^{2}$ that is not fixed at $y$, but whose position is a random vector $Y$ with density $f_{Y}$. We then define the expected number of positive parity images in $D$ as follows:

$$
E\left[N_{+}(D, Y)\right] \equiv \int_{\mathbb{R}^{2}} E\left[N_{+}(D, y)\right] f_{Y}(y) \mathrm{d} y .
$$

When the pdf $f_{Y}$ of $Y$ has compact support $\mathfrak{S}_{0}$, we write the pdf as $f_{Y, \mathfrak{S}_{0}}$ to emphasize the support and denote the expectation (15) by, 


$$
E\left[N_{+}\left(D, Y ; \mathfrak{S}_{0}\right)\right] \equiv \int_{\mathbb{R}^{2}} E\left[N_{+}(D, y)\right] f_{Y, \mathfrak{S}_{0}}(y) \mathrm{d} y .
$$

We have the following.

Corollary 7: Let $Y$ be the random source position having a general pdf $f_{Y, \mathfrak{S}_{0}}$ with compact support $\mathfrak{S}_{0}$. Then, under the assumptions of Theorem 4 , we obtain

$$
E\left[N_{+}\left(D, Y ; \mathfrak{S}_{0}\right)\right]=\int_{D} E\left[\left((1-\kappa(x))^{2}-\Gamma^{2}(x)\right) \mathbf{1}_{\mathcal{G}_{A}}(x) f_{Y, \mathfrak{S}_{0}}(\eta(x))\right] \mathrm{d} x .
$$

Proof: The result follows from Eq. (12) with $\rho=f_{Y, \mathfrak{S}_{0}}$.

Remark: It is important to add that the general formula in (16) does not require knowing the distribution of the pair $\left(\Gamma_{1}, \Gamma_{2}\right)$ conditioned on $\eta=\left(\eta_{1}, \eta_{2}\right)$, as is the case for Eq. (11). However, Eq. (16) requires knowledge of the joint distribution of the quadruple $\left(\Gamma_{1}, \Gamma_{2}, \eta_{1}, \eta_{2}\right)$. However, as will be seen in Sec. V, knowledge of the joint distribution of the pair $\left(\Gamma_{1}, \Gamma_{2}\right)$ will be enough for a certain microlensing case of interest to us.

Example: Suppose that $Y$ is uniformly distributed with pdf $f_{Y, \mathfrak{S}_{0}}$, where the compact support $\mathfrak{S}_{0}$ is a subset of the light source plane with nontrivial area,

$$
\operatorname{area}\left(\mathfrak{S}_{0}\right) \equiv\left|\mathfrak{S}_{0}\right|>0
$$

Then,

$$
E\left[N_{+}\left(D, Y ; \mathfrak{S}_{0}\right)\right]=\frac{1}{\left|\mathfrak{S}_{0}\right|} \int_{D} E\left[\left((1-\kappa(x))^{2}-\Gamma^{2}(x)\right) \mathbf{1}_{\mathcal{G}_{A}}(x) \mathbf{1}_{\mathfrak{S}_{0}}(\eta(x))\right] \mathrm{d} x .
$$

We are now ready to state the generalization of expectation from the set $\mathfrak{S}_{0}$ with positive measure to a family of sets. Let $\{\mathfrak{S}\}$ be a countable family of compact subsets with positive measure covering the light source plane $\mathbb{R}^{2}$. Suppose that each member $\mathfrak{S}$ has the same area as a fixed member $\mathfrak{S}_{0}$. For each $\mathfrak{S}$, consider random source positions $Y$ that are uniformly distributed with pdf $f_{Y, \mathfrak{S}}$ compactly supported in $\mathfrak{S}$. Note that by construction, we have

$$
f_{Y, \mathfrak{S}}(y)=\frac{\mathbf{1}_{\mathfrak{S}}(y)}{|\mathfrak{S}|}=\frac{\mathbf{1}_{\mathfrak{S}}(y)}{\left|\mathfrak{S}_{0}\right|} .
$$

Now, the quantity $E\left[N_{+}(D, Y ; \mathfrak{S})\right]$ captures an average with respect to the randomness arising from the lensing map $\eta$ as well as an average with respect to the random source position $Y$ over $\mathfrak{S}$. We now "average" $E\left[N_{+}(D, Y ; \mathfrak{S})\right]$ over the family $\{\mathfrak{S}\}$. Explicitly, we define the global expected number of positive parity images lying in $D$ for a uniform source by

$$
\hat{E}\left[N_{+}(D, Y ; \mathfrak{S})\right]_{\{\mathfrak{S}\}} \equiv \frac{1}{\left|\mathfrak{S}_{0}\right|} \int_{D} E\left[\operatorname{det}(\operatorname{Jac} \eta)(x) \mathbf{1}_{\mathcal{G}_{A}}(x)\right] \mathrm{d} x,
$$

where $|\mathfrak{S}|=\left|\mathfrak{S}_{0}\right|$. The global expected number of minimum images $\hat{E}\left[N_{+}(D, Y ; \mathfrak{S})\right]_{\{\mathfrak{S}\}}$ is well defined (i.e., bounded) since

$$
0 \leq \operatorname{det}(\operatorname{Jac} \eta)(x) \mathbf{1}_{\mathcal{G}_{A}}(x) \leq 1
$$

and $D$ is compact. This definition captures the physical setup we need for the microlensing application in Sec. V. We shall compute (17) asymptotically to three orders in the case of microlensing. 


\section{GLOBAL EXPECTED NUMBER OF IMAGES: MICROLENSING CASE}

We now consider the case of microlensing, which consists of randomly distributed point masses with continuous matter and shear, as described in Sec. II. We shall apply the results given in Secs. II and IV to study the global expected number of minimum images in microlensing.

Consider a closed disk $D$. The random lensing map $\eta_{g}$ satisfies the assumptions made at the start of Sec. IV A and in the statement of Theorem 4. In fact,

$$
P\left[\left\{x \in D: \eta_{g}(x)=y \text { and } \operatorname{det}\left(\operatorname{Jac} \eta_{g}\right)(x)=0\right\}\right]=0
$$

(i.e., lensed images in $D$ are almost surely not images of caustic points) and

$$
P[\partial D \cap\{x \in D: \eta(x)=y\}=\emptyset]=1
$$

(i.e., there is almost surely no image on the boundary of $D$ ) since there are finitely many lensed images for almost all source positions $y$ (see Ref. 6, p. 431 for more details). Note that for microlensing, we have

$$
\Gamma_{1}(x)=\gamma+\Gamma_{1, g}^{*}(x), \quad \Gamma_{2}(x)=\Gamma_{2, g}^{*}(x), \quad \text { and } \quad \kappa(x)=\kappa_{c} \quad \text { for } x \in L,
$$

where $L=\mathbb{R}^{2}-\left\{\xi_{1}, \ldots, \xi_{g}\right\}$ (lens plane) and that $D$ may contain singularities $\xi_{i}$.

Assume that $\kappa_{c}<1$ (subcritical) in this section. This implies that $N_{\max }=0$ (see Ref. 6, p. 433). Consequently, for the microlensing map $\eta_{g}$ with $\kappa_{c}<1$, we have the following for any noncaustic source position $y$,

$$
N_{+}(D, y)=N_{\min }(D, y) \equiv N_{g, \min }(D, y) .
$$

Furthermore, the set $\mathcal{G}_{A}=\left\{\nu \in \mathrm{R}^{2}: \operatorname{det}\left(\operatorname{Jac} \eta_{g}\right)(\nu) \in(0, \infty)\right\}$, when $\kappa_{c}<1$, becomes

$$
\begin{aligned}
\mathcal{G}_{A}= & \left\{\nu \in \mathbb{R}^{2}:\left(\gamma+\Gamma_{1, g}^{*}(\nu)\right)^{2}+\left(\Gamma_{2, g}^{*}(\nu)\right)^{2}<\left(1-\kappa_{c}\right)^{2}\right\}=\left\{\nu \in \mathbb{R}^{2}:\left(\Gamma_{1, g}^{*}(\nu), \Gamma_{2, g}^{*}(\nu)\right) \in B((-\gamma, 0), 1\right. \\
& \left.\left.-\kappa_{c}\right)\right\} .
\end{aligned}
$$

We are interested in determining the global expected number of minimum images in $D$, namely, we shall compute (17) for microlensing. By (17), the global expected number of minimum images would then be obtained by averaging over the possible uniform star positions (i.e., the randomness arising from the lensing map), followed by averaging the source position over a compact set with positive measure $\mathfrak{S}$, and then averaging over the possible sets in $\{\mathfrak{S}\}$, where each has the same area.

We obtain the following.

Theorem 8: Let $D \subset \mathbb{R}^{2}$ be compact and suppose that $\kappa_{c}<1$. Then, the global mean number of minimum images lying inside $D$ is given by

$$
\begin{aligned}
\hat{E}\left[N_{g, \min }(D, Y ; \mathfrak{S})\right]_{\{\mathfrak{S}\}}= & \frac{\kappa_{*} \mu_{D, \mathfrak{S}_{0}}}{2 \pi} \int_{\mathcal{B}} \frac{\left(1-\kappa_{c}\right)^{2}-(\gamma+z)^{2}-w^{2}}{\left(\kappa_{*}^{2}+z^{2}+w^{2}\right)^{3 / 2}}\left[1+\frac{H_{1}(z, w)}{g}+\frac{H_{3}(z, w)}{g^{2}}\right] \mathrm{d} z \mathrm{~d} w \\
& +O\left(g^{-3}\right),
\end{aligned}
$$

in the large $g$ limit, where

$$
\mathcal{B}=B\left((-\gamma, 0), 1-\kappa_{c}\right), \quad \mu_{D, \mathfrak{S}_{0}}=\frac{|D|}{\left|\mathfrak{S}_{0}\right|}, \quad H_{3}(z, w)=H_{2}\left(z, w ; a_{0}\right), \quad a_{0} \equiv \frac{1}{|D|} \int_{D}|x|^{2} \mathrm{~d} x,
$$

and $H_{1}$ and $H_{2}$ are defined in Eqs. (3) and (4). Recall that $|\mathfrak{S}|=\left|\mathfrak{S}_{0}\right|$ by definition of the global expectation.

Note that $\hat{E}\left[N_{g, \min }(D, Y ; \mathfrak{S})\right]_{\{\mathfrak{S}\}}$ depends on the common mass $m$ since $H_{2}$ is a function of $m$. Also the integral on the RHS of Eq. (18) remains bounded independently of $D$ although $a_{0}$ can be arbitrarily large. 
Proof: By definition,

$$
\hat{E}\left[N_{g, \min }(D, Y ; \mathfrak{S})\right]_{\{\mathfrak{S}\}}=\frac{1}{\left|\mathfrak{S}_{0}\right|} \int_{D} E\left[\operatorname{det}\left(\operatorname{Jac} \eta_{g}\right)(x) \mathbf{1}_{\mathcal{G}_{A}}(x)\right] \mathrm{d} x .
$$

Using Theorem 1, the integrand in the RHS of the above equation, which is bounded, can be written in the microlensing case as

$$
\begin{aligned}
E\left[\operatorname{det}\left(\operatorname{Jac} \eta_{g}\right)(x) \mathbf{1}_{\mathcal{G}_{A}}\right] & =E\left[\left(\left(1-\kappa_{c}\right)^{2}-\left(\gamma+\Gamma_{1, g}^{*}(x)\right)^{2}-\left(\Gamma_{1, g}^{*}\right)^{2}(x)\right) \mathbf{1}_{\mathcal{G}_{A}}(x)\right] \\
& =\int_{\mathcal{B}}\left(\left(1-\kappa_{c}\right)^{2}-(\gamma+z)^{2}-w^{2}\right) f_{\Gamma_{1, g}^{*}(x), \Gamma_{2, g}^{*}(x)}(z, w) \mathrm{d} z \mathrm{~d} w \\
& =\frac{\kappa_{*}}{2 \pi} \int_{\mathcal{B}} \frac{\left(1-\kappa_{c}\right)^{2}-(\gamma+z)^{2}-w^{2}}{\left(\kappa_{*}^{2}+z^{2}+w^{2}\right)^{3 / 2}}\left[1+\frac{H_{1}(\Gamma)}{g}+\frac{H_{2}(\Gamma ;|x|)}{g^{2}}\right] \mathrm{d} z \mathrm{~d} w+O\left(g^{-3}\right)
\end{aligned}
$$

for large $g$. Integrating over $D$ then yields the desired result.

A physical assumption often used by astronomers is the following. On the macrolensing scale (e.g., galaxy scale), the macrolens potential is averaged over the stars' positions and the lensing map is modeled by the transformation $\eta_{\text {macro }}: D \rightarrow \mathbb{R}^{2}$ given by

$$
\eta_{\text {macro }}\left(x_{1}, x_{2}\right)=\left(\left(1-\kappa_{\mathrm{tot}}+\gamma\right) x_{1},\left(1-\kappa_{\mathrm{tot}}-\gamma\right) x_{2}\right),
$$

where $\kappa_{\mathrm{tot}}=\kappa_{*}+\kappa_{c}$. In this case, choosing $\mathfrak{S}_{0}=\eta_{\text {macro }}(D)$ gives

$$
\mu_{D, \mathfrak{S}_{0}}=\frac{|D|}{\left|\mathfrak{S}_{0}\right|}=\frac{1}{\left|\left(1-\kappa_{\mathrm{tot}}\right)^{2}-\gamma^{2}\right|} \equiv \mu_{\text {macro }},
$$

which we call the macroaverage total magnification over $D$. For the case when $\mathfrak{S}_{0}=\eta_{\text {macro }}(D)$, we define

$$
\hat{E}\left[N_{g, \min }(D)\right] \equiv \hat{E}\left[N_{g, \min }(D, Y ; \mathfrak{S})\right]_{\{\mathfrak{S}\}} .
$$

Recall that the stars positions are uniformly distributed in the disk $B(\mathbf{0}, R)$ of radius $R=\sqrt{m g / \kappa_{*}}$.

Corollary 9: Let $D_{r}$ be a closed disk of radius $r$. Assume that $\mathfrak{S}_{0}=\eta_{\text {macro }}\left(D_{r}\right)$. The global mean number of minimum images is

$$
\hat{E}\left[N_{\min }\right] \equiv \lim _{r \rightarrow \infty} \lim _{g \rightarrow \infty} \hat{E}\left[N_{g, \min }\left(D_{r}\right)\right]=\frac{\kappa_{*}}{2 \pi\left|\left(1-\kappa_{\mathrm{tot}}\right)^{2}-\gamma^{2}\right|} \int_{\mathcal{B}} \frac{\left(1-\kappa_{c}\right)^{2}-(\gamma+z)^{2}-w^{2}}{\left(\kappa_{*}^{2}+z^{2}+w^{2}\right)^{3 / 2}} \mathrm{~d} z \mathrm{~d} w,
$$

and the global mean number of images and the global mean number of saddles images grow as order $O(g)$ for large $g$, with self-evident definitions.

Proof: We have $\mu_{D, \mathfrak{S}_{0}}=\mu_{\text {macro }}$. The functions $H_{1}$ and $H_{2}$ defined by Eq. (3) are bounded over $\mathbb{R}^{2}$ and $\mathbb{R}^{2} \times[0, r]$, respectively, for a fixed $r$. Thus, the function $H_{3}$ is bounded, independent of $g$. Therefore, the integrand of the integral term in Eq. (18) is bounded when we substitute $D_{r}$ for $D$. We can then take the limit as $g \rightarrow \infty$ in Eq. (18) with $D$ replaced by $D_{r}$ and apply the dominated convergence theorem to obtain

$$
\lim _{g \rightarrow \infty} \hat{E}\left[N_{g, \min }\left(D_{r}\right)\right]=\frac{\kappa_{*}}{2 \pi\left|\left(1-\kappa_{\mathrm{tot}}\right)^{2}-\gamma^{2}\right|} \int_{\mathcal{B}} \frac{\left(1-\kappa_{c}\right)^{2}-(\gamma+z)^{2}-w^{2}}{\left(\kappa_{*}^{2}+z^{2}+w^{2}\right)^{3 / 2}} \mathrm{~d} z \mathrm{~d} w,
$$

which is independent of $D_{r}$. Taking the limit as $r \rightarrow \infty$ yields the first result of the corollary. The second one then follows from Corollary 6. 
TABLE I. Global mean number of minimum images on the lens plane (cf. Corollary 9).

\begin{tabular}{cccccc}
\hline \hline \multicolumn{7}{c}{$\hat{E}\left[N_{\text {min }}\right]$ : global mean number of minima } \\
\hline$\gamma \backslash \kappa_{*}$ & 0.1 & 0.2 & 0.3 & 0.4 & 0.45 \\
\hline 0.1 & 1.037 & 1.114 & 1.219 & 1.345 & 1.415 \\
0.2 & 1.068 & 1.171 & 1.230 & 1.448 & 1.529 \\
0.3 & 1.142 & 1.306 & 1.488 & 1.688 & 1.794 \\
0.4 & 1.349 & 1.667 & 1.985 & 2.313 & 2.484 \\
0.5 & 2.520 & 3.620 & 4.621 & 5.608 & 6.108 \\
\hline \hline
\end{tabular}

\section{Remark:}

(1) For physically relevant values of $\kappa_{*}, \kappa_{c}$, and $\gamma$, the RHS of (19) is finite. However, the global mean is divergent for $\left(1-\kappa_{\mathrm{tot}}\right)^{2}=\gamma^{2}$.

(2) This corollary agrees with the findings by Wambsganss, Witt, and Schneider ${ }^{9}$ and Granot, Schrecter, and Wambsganss. ${ }^{10}$ The integral in Eq. (19) was resolved in closed form using elliptic integrals (see Ref. 10).

In Table I, we illustrate $\hat{E}\left[N_{\min }\right]$ for $\kappa_{\mathrm{tot}}=\kappa_{c}+\kappa_{*}=0.450$ and varying $\gamma$ and $\kappa_{*}$. For all the values of $\kappa_{*}$ and $\gamma$ shown, we have the "macrominimum" situation of $1-\kappa_{\mathrm{tot}}+\gamma>0$ and $1-\kappa_{\mathrm{tot}}$ $-\gamma>0$. Note that the number of images increases significantly when $\gamma$ gets close to $1-\kappa_{\text {tot }}$. In the column for $\kappa_{*}=0.45=\kappa_{\mathrm{tot}}$, note how $\hat{E}\left[N_{\text {min }}\right]$ increases to 6.108 as $\gamma$ increases.

We see from Table I that the global mean number of minima is relatively small. For $\kappa_{\text {tot }}$ $=0.450$ and admissible values of $\gamma, \kappa_{c}$, and $\kappa_{*}$, we observe that the global mean number of minima is between 1 and 3 .

We present values of $\hat{E}\left[N_{g, \min }(D)\right]$, the global mean number of minima lying inside the disk $D=B(\mathbf{0}, 1111.11)$, in Table II. Here, we fix $g=1000, m=1$, and $\kappa_{\mathrm{tot}}=0.450$ and we vary the values of $\kappa_{*}$ and $\gamma$.

The values in Table II differ from the corresponding values in Table I by a relatively small amount. This is expected as the difference between $\hat{E}\left[N_{g, \min }(D)\right]$ and $\hat{E}\left[N_{\text {min }}\right]$ has order of $O\left(g^{-1}\right)$ (by Theorem 8 and Corollary 9).

\section{CONCLUSION}

We carried on our development of a mathematical theory for stochastic microlensing, devoting our efforts to the study of the expected number of different types of lensed images. This involved an analysis of the random microlensing shear. In particular, we obtained the first three leading terms in their asymptotic joint pdf's in the limit of an infinite number of stars and showed that each of their marginals converges to a shifted Cauchy distribution, which has a heavy tail. The

TABLE II. Asymptotics of the global mean number of minima over the disk $B(\mathbf{0}, 1111.11)$ (cf. Theorem 8).

\begin{tabular}{cccccc}
\hline \hline \multicolumn{7}{c}{$\hat{E}\left[N_{g, \min }(D)\right]:$ global mean number of minima in $D$} \\
\hline$\gamma \backslash \kappa_{*}$ & 0.1 & 0.2 & 0.3 & 0.4 & 0.45 \\
\hline 0.1 & 1.036 & 1.113 & 1.217 & 1.343 & 1.412 \\
0.2 & 1.067 & 1.170 & 1.297 & 1.446 & 1.526 \\
0.3 & 1.142 & 1.305 & 1.486 & 1.685 & 1.791 \\
0.4 & 1.349 & 1.666 & 1.982 & 2.309 & 2.479 \\
0.5 & 2.519 & 3.617 & 4.615 & 5.599 & 6.096 \\
\hline \hline
\end{tabular}


third leading order term of this joint pdf depends on the magnitude of the shear due to the stars, the optical depth, and the mean number of stars within a disk centered at the origin. We also obtained the asymptotic pdf of the shear's magnitude in the limit of an infinite number of stars. These results on the random shear are given for a general point in the lens plane. Using the Kac-Rice formula and Morse theory, we determined the expected total number of images and expected number of saddle images for a general random lensing map. ${ }^{22}$ By formulating a generalization of the Kac-Rice type formula to a family of compact subsets with positive measure of the light source plane, we introduced the global expected number of positive parity images for a general random lensing situation. Our general results are applicable to a wide range of lensing models. Specifically, we computed the global expected number of minimum images for the case of microlensing. This global expectation was also illustrated with explicit numerical values.

\section{ACKNOWLEDGMENTS}

We thank the referees for invaluable feedback that strengthened the paper. A.M.T. would like to thank A. Aazami, R. Adler, J. Mattingly, and A. Watkins for helpful discussions. A.O.P. acknowledges the support of NSF Grant Nos. DMS-0707003 and AST-0434277-02. Part of this work was done at the Petters Research Institute in Belize.

\section{APPENDIX: PROOF THAT $\mathfrak{I}_{n}=0$}

We consider the integral

$$
\mathfrak{I}_{n} \equiv \int_{R-|x|}^{R+|x|} \frac{\sin [n f(r)]}{r^{n-1}} \mathrm{~d} r
$$

for any integer $n \geq 2$ and $0 \leq|x|<R$, where

$$
f(r)=\arccos \left(\frac{|x|^{2}+r^{2}-R^{2}}{2|x| r}\right) .
$$

Define the variable $\vartheta$ by

$$
R \cos \vartheta=r \cos f(r)-|x|,
$$

with $\vartheta$ restricted to the interval $[0, \pi]$. This implies that $r \sin f(r)=R \sin \vartheta$. Therefore,

$$
r \exp [i f(r)]=|x|+R e^{i \vartheta} \quad \text { and } \quad r \exp [-i f(r)]=|x|+R e^{-i \vartheta} .
$$

We now use these equalities and the change of variable from $r$ to $\vartheta$ to obtain the following:

$$
\begin{aligned}
\mathfrak{I}_{n+1} & =\frac{1}{2 i} \int_{R-|x|}^{R+|x|}\left[\frac{e^{i(n+1) f(r)}-e^{-i(n+1) f(r)}}{r^{n}}\right] \mathrm{d} r \\
& =\frac{1}{2 i} \int_{R-|x|}^{R+|x|}\left[\frac{1}{\left(r e^{-i f(r)}\right)^{n+1}}-\frac{1}{\left(r e^{i f(r)}\right)^{n+1}}\right] r \mathrm{~d} r \\
& =\frac{R|x|}{2 i} \int_{0}^{\pi}\left[\frac{1}{\left(|x|+R e^{-i \vartheta}\right)^{n+1}}-\frac{1}{\left(|x|+R e^{i \vartheta}\right)^{n+1}}\right] \sin \vartheta \mathrm{d} \vartheta \\
& =\frac{R|x|}{2 i} \int_{-\pi}^{\pi} \frac{\sin \vartheta}{\left(|x|+R e^{-i \vartheta}\right)^{n+1}} \mathrm{~d} \vartheta \\
& =\frac{R|x|}{(2 i)^{2} R^{n+1}} \int_{-\pi}^{\pi} \frac{e^{i \vartheta}-e^{-i \vartheta}}{e^{-i(n+1) \vartheta}\left(1+\frac{|x|}{R} e^{i \vartheta}\right)^{n+1}} \mathrm{~d} \vartheta
\end{aligned}
$$




$$
\begin{aligned}
& =-\frac{|x|}{4 R^{n}} \int_{-\pi}^{\pi}\left(e^{i(n+2) \vartheta}-e^{i n \vartheta}\right)\left(1+\frac{|x|}{R} e^{i \vartheta}\right)^{-(n+1)} \mathrm{d} \vartheta \\
& =\frac{|x|}{4 R^{n}} \sum_{k=0}^{\infty} \frac{(-)^{k} \Gamma(k+n+1)}{k ! \Gamma(n+1)} \frac{|x|^{k}}{R^{k}} \int_{-\pi}^{\pi}\left(e^{i(n+k) \vartheta}-e^{i(n+2+k) \vartheta}\right) \mathrm{d} \vartheta \\
& =0 .
\end{aligned}
$$

Above, we used the inequality $0 \leq|x|<R$ to obtain the Taylor series expansion. Also, the convergence of the series allowed us to reverse the order of the summation and the integral. Finally, the last line follows by direct integration, where we used the assumption $n+1 \geq 2$, that is, $n \geq 1$.

${ }^{1}$ A. O. Petters, B. Rider, and A. M. Teguia, J. Math. Phys. 50, 072503 (2009).

${ }^{2}$ A. Einstein, Science 84, 506 (1936).

${ }^{3}$ A. O. Petters, J. Math. Phys. 33, 1915 (1992).

${ }^{4}$ A. O. Petters, J. Math. Phys. 36, 4276 (1995).

${ }^{5}$ A. O. Petters, J. Math. Phys. 38, 1605 (1997).

${ }^{6}$ A. O. Petters, H. Levine, and J. Wambsganss, Singularity Theory and Gravitational Lensing (Birkhauser, Boston, 2001).

${ }^{7}$ S. H. Rhie, e-print arXiv:astro-ph/0305166.

${ }^{8}$ D. Khavinson and G. Neumann, Proc. Am. Math. Soc. 134, 1077 (2006).

${ }^{9}$ J. Wambsganss, H. J. Witt, and P. Schneider, Astron. Astrophys. 258, 591 (1992).

${ }^{10}$ J. Granot, P. L. Schrecter, and J. Wambsganss, Astrophys. J. 583, 575 (2003).

${ }^{11}$ R. Adler and J. Taylor, Random Fields and Geometry (Wiley, London, 1981).

${ }^{12}$ J. M. Azaïs and M. Wschebor, Ann. Appl. Probab. 15, 254 (2005).

${ }^{13}$ P. J. Forrester and G. Honner, J. Phys. A 32, 2961 (1999).

${ }^{14}$ M. Sodin and B. Tsirelson, Isr. J. Math. 144, 125 (2004).

${ }^{15}$ M. Sodin and B. Tsirelson, Isr. J. Math. 152, 105 (2006).

${ }^{16}$ M. Sodin and B. Tsirelson, Isr. J. Math. 147, 371 (2005).

${ }^{17}$ L. Shepp and R. J. Vanderbei, Trans. Am. Math. Soc. 347, 4365 (1995).

${ }^{18}$ J. Milnor, Morse Theory (Princeton University Press, Princeton, 1963).

${ }^{19}$ R. Nityananda and J. P. Ostriker, J. Astrophys. Astron. 5, 235 (1984).

${ }^{20}$ P. Schneider, J. Ehlers, and E. Falco, Gravitational Lenses (Springer, Berlin, 1992).

${ }^{21}$ W. Rudin, Real and Complex Analysis (McGraw-Hill, New York, 1987).

${ }^{22}$ H. Federer, Geometric Measure Theory (Springer, Berlin, 1969). 